\title{
Type IV fimbrial subunit protein ApfA contributes to protection against porcine pleuropneumonia
}

\author{
Lenka Sadilkova', Jiri Nepereny ${ }^{2}$, Vladimir Vrzal ${ }^{2}$, Peter Sebo ${ }^{1}$ and Radim Osicka ${ }^{1 *}$
}

\begin{abstract}
Porcine pleuropneumonia caused by Actinobacillus pleuropneumoniae accounts for serious economic losses in the pig farming industry worldwide. We examined here the immunogenicity and protective efficacy of the recombinant type IV fimbrial subunit protein ApfA as a single antigen vaccine against pleuropneumonia, or as a component of a multi-antigen preparation comprising five other recombinant antigens derived from key virulence factors of A. pleuropneumoniae (ApxIA, ApxIIA, ApxIIIA, ApxIVA and TbpB). Immunization of pigs with recombinant ApfA alone induced high levels of specific serum antibodies and provided partial protection against challenge with the heterologous A. pleuropneumoniae serotype 9 strain. This protection was higher than that engendered by vaccination with rApxIVA or rTbpB alone and similar to that observed after immunization with the tri-antigen combination of rApxIA, rApxIIA and rApxIIIA. In addition, rApfA improved the vaccination potential of the pentaantigen mixture of rApxIA, rApxIIA, rApxIIIA, rApxIVA and rTbpB proteins, where the hexa-antigen vaccine containing rApfA conferred a high level of protection on pigs against the disease. Moreover, when rApfA was used for vaccination alone or in combination with other antigens, such immunization reduced the number of pigs colonized with the challenge strain. These results indicate that ApfA could be a valuable component of an efficient subunit vaccine for the prevention of porcine pleuropneumonia.
\end{abstract}

\section{Introduction}

Actinobacillus pleuropneumoniae, the etiological agent of porcine pleuropneumonia, is a Gram-negative bacterium colonizing the porcine respiratory tract [1-3]. Pleuropneumonia is a severe contagious and economically significant disease. It can range from acute to chronic, depending on host age, immune status, the bacterial strain causing the infection, or the infective dose [4-6]. The acute stage is characterized by a haemorrhagic necrotizing pneumonia and fibrinous pleuritis and may progress rapidly to death $[7,8]$. In the chronic stage, localized lung lesions and adhesive pleuritis can be observed and chronically infected animals can become a source of infection for the whole non-infected herd $[1,2,9]$.

To control porcine pleuropneumonia, vaccination is useful $[10,11]$, but development of efficient vaccines against the disease appears difficult due to the existence and diversity of 15 serotypes of A. pleuropneumoniae that are differentiated on the basis of surface polysaccharide

\footnotetext{
* Correspondence: osicka@biomed.cas.cz

'Institute of Microbiology of the Academy of Sciences of the Czech

Republic, v.vi.i, Videnska 1083, CZ-142 20 Prague, Czech Republic

Full list of author information is available at the end of the article
}

antigens [12-14]. The first vaccines against $A$. pleuropneumoniae infection comprised formalin-treated or heat-inactivated bacteria. These whole-cell bacterin vaccines reduce mortality after challenge with the homologous serotypes of $A$. pleuropneumoniae, but usually do not confer efficient protection against infection with heterologous serotypes [15-17]. The limited protection observed with bacterins might be explained by (i) the absence of secreted immunogenic proteins, such as the ApxA toxins that are the key virulence factors of $A$. pleuropneumoniae, (ii) the alteration of antigenic potency of certain bacterial antigens due to inactivation treatment, or (iii) the absence of immunogenic antigens that are expressed only within the host [11,18-22]. Indeed, pigs surviving natural or experimental infection with $A$. pleuropneumoniae were found to be completely protected against homologous serotypes and generally also against heterologous serotype infections $[16,23,24]$. To overcome the drawbacks of bacterins, live attenuated vaccines that reflect natural $A$. pleuropneumoniae infection and allow the in-vivo production of immunogenic antigens were developed, comprising temperature-sensitive, streptomycin-dependent or metabolic mutants, or mutants having deleted or inactivated genes for key virulence factors
Ciomed Central

() 2012 Sadilkova et al; licensee BioMed Central Ltd. This is an Open Access article distributed under the terms of the Creative Commons Attribution License (http://creativecommons.org/licenses/by/2.0), which permits unrestricted use, distribution, and reproduction in any medium, provided the original work is properly cited. 
[25-34]. Some of the live attenuated vaccines tested indeed confer a high-level cross-protection in contrast to wholecell bacterin vaccines $[30,35,36]$. Despite many promising results, the use of live bacteria brings numerous safety drawbacks that could be eliminated by the development of a highly efficient subunit vaccine. Among the valuable components of different subunit vaccines, the key virulence factors of A. pleuropneumoniae, such as the ApxA exotoxins, the outer membrane proteins, or iron-acquisition factors, were tested, respectively [11].

A. pleuropneumoniae secretes three different ApxA exotoxins (ApxIA, ApxIIA, and ApxIIIA), which are members of the RTX (Repeat in ToXin) family [3,37-42]. ApxIA shows strong hemolytic activity, while ApxIIA exhibits weaker hemolytic activity $[43,44]$. Both are cytotoxic and active on a broad range of cells of different types and species [45]. ApxIIIA is nonhemolytic, but it is strongly cytotoxic and targets mainly porcine alveolar macrophages and neutrophils $[44,46]$. The ApxA exotoxins are thought to be of particular importance as antigens for inducing protective immunity against pleuropneumonia and have been included in a broad range of tested subunit vaccines [21,47-49]. A fourth secreted RTX protein of $A$. pleuropneumoniae, ApxIVA, was described and its biologic activity remains unknown [50]. ApxIVA appears to be produced in vivo but not under in vitro conditions $[19,50]$. Recently, the contribution of recombinant ApxIVA to the protective efficacy of a subunit vaccine against A. pleuropneumoniae challenge was demonstrated [49].

To survive in the iron-depleted environment of the host, A. pleuropneumoniae produces the surface proteins, TbpA and $\mathrm{TbpB}$ (transferrin binding proteins), that enable the pathogen to utilize porcine transferrin as a source of iron [51]. Both transferrin binding proteins are key virulence factors of A. pleuropneumoniae [52]. It has been shown that pigs immunized with recombinant $\mathrm{TbpB}$ are less susceptible to the disease after experimental homologous challenge with $A$. pleuropneumoniae serotype 7 [53].

Since each of the $A$. pleuropneumoniae proteins alone offers only a partial protection against porcine pleuropneumonia, current commercially available or newly tested vaccines consist of combinations of several bacterial antigens $[21,47-49,53]$. These subunit vaccines are effective at preventing acute disease, but do not sufficiently protect against colonization and are not widely cross-protective $[33,47]$. Therefore, further A. pleuropneumoniae outer membrane and/or secreted proteins are screened and tested as candidates for inclusion into next generation vaccines [54]. Among the promising antigens to be included in a subunit vaccine against porcine pleuropneumonia is the type IV fimbrial subunit protein. Type IV fimbriae (pili) are typically composed of thousands of subunit proteins polymerized into a fiber that protrudes from the bacterial cell surface [55]. Fimbriae are, indeed, known to play an important role in the pathogenesis of Gram-negative bacteria such as Moraxella catarrhalis, Neisseria meningitidis, N. gonorrhoeae, Haemophilus influenzae, enteropathogenic Escherichia coli or Pseudomonas aeruginosa and are involved in adherence and colonization, twitching motility, biofilm formation, protein export and DNA uptake [56-63]. Type IV fimbriae were also isolated from $A$. pleuropneumoniae and a 15.9$\mathrm{kDa}$ ApfA protein was identified as the type IV fimbrial subunit protein [55]. The apfA gene was found to be present in 12 representative $A$. pleuropneumoniae serotype strains and exhibited greater than $98 \%$ identity on DNA level with the predicted amino acid sequences being $100 \%$ identical [64]. Moreover, all typical fimbrial biogenesis genes were identified in A. pleuropneumoniae and appear to form a type IV fimbrial operon with apfA [18]. This is present in a single copy in the A. pleuropneumoniae genome and was found to be expressed in vivo, but not in vitro [18]. The single copy and high conservation of the apfA gene, the surface location of the ApfA protein and its putative role in adherence, colonization and other important biological functions, make ApfA a particularly attractive target for vaccine development.

Here, we tested the immunogenicity and protective potential of recombinant ApfA against porcine pleuropneumonia, either in a single antigen vaccine, or as a component of a multi-antigen preparation, including five other purified recombinant antigens derived from key virulence factors of $A$. pleuropneumoniae.

\section{Materials and methods}

\section{Bacterial strains and growth conditions}

The E. coli K12 strain, XL1-Blue (Stratagene, La Jolla, USA), was used throughout this work for DNA manipulations and was grown in Luria-Bertani (LB) medium supplemented with $60 \mu \mathrm{g} / \mathrm{mL}$ of kanamycin. The $E$. coli BL21( $\lambda$ DE3) strain (Invitrogene, Carlsbad, USA) was used for expression of recombinant proteins and was grown at $37^{\circ} \mathrm{C}$ in $\mathrm{LB}$ medium containing $60 \mu \mathrm{g} / \mathrm{mL}$ of kanamycin.

A. pleuropneumoniae serotypes 1, 2, 3 and 7 were used for isolation of genomic DNA and A. pleuropneumoniae serotype 9 strain was used for challenge experiments. A. pleuropneumoniae serotype 7 strain was obtained from the Veterinary Research Institute, Czech Republic - Collection of animal pathogenic microorganisms (CAPM 3800 serovar 7, strain: WF 83, S. Rosendal, Canada, source: pig/lung (pleuropneumonia)). All other serotypes of A. pleuropneumoniae were isolated in the Moravske Prusy agricultural co-operative (Czech Republic) from the lungs of pigs with respiratory infection (after necropsy). It was verified by PCR-cloning and DNA sequencing that the serotype 9 strain used for challenge experiments harbored a fully conserved apfA gene allele. 
A. pleuropneumoniae was grown on Columbia agar base (Lab M, Bury, UK) supplemented with 10\% NAD (SigmaAldrich, St. Louis, USA) for $24 \mathrm{~h}$ at $37^{\circ} \mathrm{C}$ in $5 \% \mathrm{CO}_{2}$ and then inoculated into the liquid culture (Supplemented Columbia broth (BBL Microbiology Systems, Cockeysville, USA)) supplemented with $1 \%$ IsoVitaleX (BBL Microbiology Systems, Cockeysville, USA) and $10 \mu \mathrm{g} / \mathrm{mL}$ of NAD, grown for $6 \mathrm{~h}$ and harvested by centrifugation at $4500 \times g$ for $15 \mathrm{~min}$.

\section{Isolation and cloning of genes encoding ApfA, ApxA and TbpB proteins}

The A. pleuropneumoniae genomic DNA was isolated from serotypes 1, 2, 3 and 7 using phenol-chloroform extraction [65] and the apfA, apxIA, apxIIA, apxIIIA, apxIVA and $t b p B$ genes were amplified by polymerase chain reaction (PCR) (Table 1). Reaction mixtures $(70 \mu \mathrm{L})$ contained 100 ng of genomic DNA, $2 \mu \mathrm{M}$ of each oligonucleotide primer, $100 \mathrm{mM} \mathrm{KCl,} 20 \mathrm{mM}$ Tris- $\mathrm{HCl}$ (pH 8.3), $7.5 \mathrm{mM} \mathrm{MgCl}_{2}$, $1 \mathrm{mM}$ DTT, $200 \mu \mathrm{g} / \mathrm{mL}$ BSA, $140 \mu \mathrm{M}$ of each dNTP and 1 unit of Phusion DNA polymerase (Finnzymes, Espoo, Finland). Reaction mixtures were pre-heated to $98^{\circ} \mathrm{C}$ for $30 \mathrm{~s}$ and subjected to 30 cycles of $98^{\circ} \mathrm{C}$ for $10 \mathrm{~s}, 52^{\circ} \mathrm{C}$ for $30 \mathrm{~s}$, and $72^{\circ} \mathrm{C}$ for $3 \mathrm{~min}$ using a PCR Sprint instrument (ThermoHybaid, Middlesex, UK). Specific primer pairs for PCR amplification were designed based on the published sequences of the apfA, apxIA, apxIIA, apxIIIA, apxIVA and $t b p B$ genes (Table 1) $[37,66,67]$. To facilitate cloning, the primers were designed to produce a PCR product with a restriction site for the Sal I enzyme at the 5'-end and for Not I at the 3'-end, respectively (Table 1). PCR products were isolated from agarose gel, digested with the Sal I and Not I restriction enzymes and fused in frame with the sequence for $6 \times$ His tag at the 5'- and 3'-ends by cloning into the Sal I and Not I sites of the modified pET28b_Nco$\mathrm{Sal}$ vector. This construct was prepared by ligation of Nco
I - Sal I digested pET28b (Novagen, Madison, USA) with a double-stranded adapter (5'-CATGCATCATCATCATCATCATCATAG-3' and 5'-TCGACTATGATGATGATGATGATGATG-3'). The set of expression vectors, pET28b-apfA, pET28b-apxIA, pET28b-apxIIA, pET28b$a p x I I I A, \mathrm{pET} 28 \mathrm{~b}-a p x I V A$ and $\mathrm{pET} 28 \mathrm{~b}-t b p B$, was verified by restriction analysis and DNA sequencing.

\section{Protein production and purification}

All recombinant proteins were produced in $E$. coli strain BL21( $\lambda$ DE3) transformed with the appropriate plasmid. Exponential $500 \mathrm{~mL}$ cultures grown with shaking at $37^{\circ} \mathrm{C}$ in LB medium supplemented with $60 \mu \mathrm{g} / \mathrm{mL}$ of kanamycin were induced at $\mathrm{OD}_{600}=0.6-0.8$ with $1 \mathrm{mM}$ IPTG and grown for an additional $4 \mathrm{~h}$. The cells were harvested by centrifugation, washed twice with $50 \mathrm{mM}$ Tris- $\mathrm{HCl}(\mathrm{pH}$ 8.0), $150 \mathrm{mM} \mathrm{NaCl}$ (TN buffer) and disrupted by sonication at $4{ }^{\circ} \mathrm{C}$. The extracts were cleared at $20000 \times g$ for $20 \mathrm{~min}$, supernatants were discarded and pellets were solubilized with $50 \mathrm{mM}$ Tris- $\mathrm{HCl}$ ( $\mathrm{pH} \mathrm{8.0),} 8 \mathrm{M}$ urea and $300 \mathrm{mM} \mathrm{NaCl}$ (TUN buffer) and centrifuged at $20000 \times g$ for additional $20 \mathrm{~min}$. The obtained urea extracts were loaded on a Ni-NTA agarose column (Qiagen, Valencia, USA) equilibrated with the TUN buffer. Washing was performed with equilibration buffer and recombinant proteins were eluted with TUN buffer supplemented with $250 \mathrm{mM}$ imidazole. To remove the imidazole from the samples, the most concentrated fractions were loaded on Sephadex G25 (Sigma-Aldrich, St. Louis, USA) and transferred into the TUN buffer.

\section{DNA manipulations and recombinant protein analysis} SDS polyacrylamide gel electrophoresis (SDS-PAGE), Western blot analysis, determination of protein concentrations and in vitro DNA manipulations were performed according to standard protocols [65].

Table 1 PCR primer pairs, origin and features of $a p f A$, apxA and $t b p B$ genes and corresponding proteins

\begin{tabular}{|c|c|c|c|c|}
\hline Gene $^{1}$ & $\begin{array}{l}\text { A. pleuropneumoniae } \\
\text { serotype }\end{array}$ & PCR primer pair $\left(5^{\prime} \rightarrow 3^{\prime}\right)^{2}$ & $\begin{array}{l}\text { Gene length } \\
\text { (bps) }\end{array}$ & $\begin{array}{l}\text { Protein MW } \\
(\mathrm{kDa})^{3}\end{array}$ \\
\hline apfA & 7 & $\begin{array}{l}\text { CCGTCGACATGTCATATAACAGTTATAC } \\
\text { CCGCGGCCGCATTTGATGCGCAGAAATTT }\end{array}$ & 342 & 14 \\
\hline$a p x I A$ & 1 & $\begin{array}{l}\text { GGGTCGACACGCTAACTCTCAGCTCGATA } \\
\text { GGGCGGCCGCAGCTGCTTGTGCTAAAGAA }\end{array}$ & 3069 & 113 \\
\hline apxlIA & 7 & $\begin{array}{l}\text { CCGTCGACACTCAAAAATCACTTTGTCATCA } \\
\text { C(ECGGCCGCAGCGGCTCTAGCTAATTG }\end{array}$ & 2871 & 105 \\
\hline apxIIIA & 2 & $\begin{array}{l}\text { GGGTCGACACAGTACTTGGTCAAGCATGTT } \\
\text { GGGCGGCCGCAGCTGCTCTAGCTAGGTTA }\end{array}$ & 3159 & 115 \\
\hline apxIVA & 3 & $\begin{array}{l}\text { CCGTCGACACACAAAATTAACTATGCAAGA } \\
\text { CCGCGGCCGCTAAAGCAGCTGTTAAGCTATT }\end{array}$ & 5418 & 205 \\
\hline tbp B & 7 & 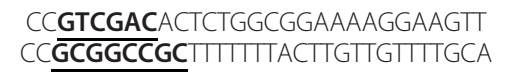 & 1644 & 60 \\
\hline
\end{tabular}

${ }^{1}$ Sequences of genes were acquired from GenBank (apfA, AY235719.1; apxIA, AF240779; apxIIA, CP001091; apxIIIA, L12145; apxIVA, AF030511; tbpB, U16017). ${ }^{2}$ All oligonucleotides were synthesized by Generi Biotech, Hradec Kralove, Czech Republic. First oligonucleotide represents forward PCR primer, second reverse PCR primer. Sal I and Not I recognition sites are marked in bold and underlined.

${ }^{3}$ The approximate protein molecular weight was calculated using the Compute pl/Mw tool (ExPASy). 


\section{Preparation of A. pleuropneumoniae for challenge experiments}

A. pleuropneumoniae serotype 9 strain was grown to $\mathrm{OD}_{600}=0.6$, culture was centrifuged at $4500 \times g$ for $15 \mathrm{~min}$ and resuspended in sterile PBS (phosphate-buffered saline, $\mathrm{pH}$ 7.4). The suspension was plated in triplicate in 10-fold serial dilutions onto Columbia agar supplemented with $10 \% \mathrm{NAD}$ and grown for $24 \mathrm{~h}$ at $37^{\circ} \mathrm{C}$ in $5 \% \mathrm{CO}_{2}$ for the exact estimation of the number of colony-forming units (CFU). The bacteria used for challenge experiments were tested by Western blot for the production of ApxIA and ApxIIA toxins that are known to be of major importance for the full virulence of A. pleuropneumoniae. The challenge suspension was administered to experimental animals using an intranasal probe.

\section{Experimental animals}

For the immunization and challenge experiments, 8-week old piglets (Agricultural Co-operative, Tesnovice, Czech Republic) of both genders, cross-breeds of Czech White and Landrace, weighting 21-24 kg, were used. All piglets were tested prior to vaccination for the absence of serum antibodies against A. pleuropneumoniae (Chekit APPApxIV, IDEXX Laboratories, Westbrook, USA), Pasteurella multocida (PMT ELISA Kit, DakoCytomation, Glostrup, Denmark), Mycoplasma hyopneumoniae (HerdChek M. hyo, IDEXX), Salmonella spp. (HerdChek Swine Salmonella, IDEXX), porcine reproductive and respiratory syndrome virus (IDEXX PRRS X3, IDEXX), swine influenza virus (CIVTEST Suis Influenza, Hipra Laboratories, Gerona, Spain) and pseudorabies virus (HerdChek AntiPRV gB, IDEXX), respectively. None of the piglets showed disease signs before or at the time of challenge. Handling with animals was performed according to the national legislation on animal welfare and in line with internal guidelines of Bioveta, a. s. (SOPs of Bioveta and the Guidelines for the Care and Use of Laboratory Animals, the Act of the Czech National Assembly, Collection of laws No. 149/ 2004, inclusive of the amendments, on the Protection of Animals against Cruelty, and Public Notice of the Ministry of Agriculture of the Czech Republic, Collection of laws No. 207/2004, on keeping and exploitation of experimental animals).

\section{Determination of $A$. pleuropneumoniae challenge dose in pigs}

The challenge dose for the intranasal infection, eliciting overt signs of severe porcine pleuropneumonia and causing mortality in $50 \%$ of animals (LD50), was determined for a local A. pleuropneumoniae serotype 9 strain. Three groups of four pigs were inoculated intranasally with $3 \times$ $10^{6}, 1 \times 10^{7}$ and $2 \times 10^{8} \mathrm{CFU}$ (each $5 \mathrm{~mL}$ dose was split into two $2.5 \mathrm{~mL}$ aliquots and applied to both nostrils) of bacteria and the pigs were monitored daily for 4 days for signs of morbidity and mortality rate. Two out of four challenged pigs died within 24 hours from challenge with the dose of $2 \times 10^{8} \mathrm{CFU}$, which was used further as the challenge dose in tests of vaccine potency.

\section{Immunizations and challenge experiments in pigs}

The immunization and challenge experiments were performed twice and the results obtained exhibited a good concordance. In total, twenty-five pigs in experiment 1 and nineteen pigs in experiment 2 , respectively, were randomly allocated into 8 groups (Table 2) and sampled to obtain preimmune sera. Animals in groups 1 to 6 were intramuscularly injected with purified recombinant antigens in oil adjuvant (antigen(s) diluted in $1.5 \mathrm{~mL}$ of PBS to a final amount indicated in the text below plus $0.5 \mathrm{~mL}$ of EMULSIGEN, MVP laboratories, Inc., USA) as follows: Group 1) 12 pigs, immunized with rApfA (100 $\mu \mathrm{g}$ per dose); group 2) 3 pigs, injected with rApxIVA (100 $\mu$ g per dose); group 3) 3 pigs, immunized with $\mathrm{rTbpB}(100 \mu \mathrm{g}$ per dose); group 4) 3 pigs, immunized with a combination of rApxIA, rApxIIA and rApxIIIA (50 $\mu$ g each per dose); group 5) 7 pigs, injected with a penta-antigen combination of rApxIA, rApxIIA, rApxIIIA, rApxIVA and rTbpB proteins $(50 \mu \mathrm{g}$ each per dose); group 6$) 7$ pigs, immunized with a hexa-antigen vaccine including $\mathrm{rApfA}(100 \mu \mathrm{g}$ per dose) and rApxIA, rApxIIA, rApxIIIA, rApxIVA, rTbpB (50 $\mu \mathrm{g}$ each per dose). Two weeks later all the groups were revaccinated with the same dose of antigens. Seven pigs in group 7 received PBS in oil adjuvant and 2 pigs in group 8 were left non-vaccinated. The post-vaccination sera from all piglets were collected one day before challenge and the levels of antibodies against the antigens were tested by ELISA. Animals in groups 1 to 7 were intranasally challenged one month after the first immunization. Inocula were applied using an intranasal probe and consisted of the $\mathrm{LD}_{50}$ infectious dose of $A$. pleuropneumoniae serotype 9 strain $\left(2 \times 10^{8} \mathrm{CFU}\right.$ in $\left.5 \mathrm{~mL}\right)$. Following challenge, piglets were examined and scored daily over seven days using a 0 to 4 scale: 0 , no signs of porcine pleuropneumonia; 1 , increased respiration rate, and/or sporadic coughing, and/ or occasional lying down, and/or mild apathy; 2 , abdominal respiration, and/or coughing, and/or lying down, and/ or apathy; 3, dyspnea, and/or regular coughing, and/or lying down, and/or markedly depressed appearance, and/ or bloody discharge, and/or agony; 4, death. Moreover, body temperature was taken daily. On day 7 post-challenge all surviving pigs were sacrificed and their lungs were subjected to examination for lesions. These were scored as previously described $[49,68]$ with the following modifications: 0 , no pathological changes; 1 , minor pathological changes, $1-20 \%$ of the lobe affected; 2 , larger damage of the lobe, $21-50 \%$ of the lobe affected; 3 , severe damage of the lobe, $51-100 \%$ of the lobe affected. The left and right cranial, middle and caudal 
Table 2 Clinical score, lung lesion score and re-isolation of the challenge strain after immunization of pigs with different vaccines, followed by heterologous intranasal challenge with $A$. pleuropneumoniae

\begin{tabular}{|c|c|c|c|c|c|c|}
\hline Vaccine $^{1}$ & $\begin{array}{l}\text { Number of } \\
\text { pigs }^{2}\end{array}$ & $\begin{array}{l}\text { Clinical } \\
\text { score }^{3}\end{array}$ & $\begin{array}{c}\text { Body } \\
\text { temperature }\left({ }^{\circ} \mathrm{C}\right)\end{array}$ & $\begin{array}{l}\text { Lung lesion } \\
\text { score }^{5}\end{array}$ & $\begin{array}{l}\text { Re-isolation of the challenge } \\
\text { strain }(\%)^{6}\end{array}$ & $\begin{array}{l}\text { Surviving } \\
\text { pigs (\%) }\end{array}$ \\
\hline rApfA & 12 & $1.7 \pm 0.7^{7}$ & $40.3 \pm 0.5^{7}$ & $1.3 \pm 0.7^{7}$ & $58^{7}$ & 100 \\
\hline rApxIVA & 3 & $2.6 \pm 0.5$ & $40.8 \pm 0.4$ & $1.8 \pm 0.5$ & 100 & 100 \\
\hline rTbpB & 3 & $2.7 \pm 0.5$ & $40.9 \pm 0.5$ & $1.9 \pm 0.7$ & 100 & 100 \\
\hline rApxIA+rApxIIA+rApxIIIA & 3 & $1.9 \pm 0.7$ & $40.5 \pm 0.4$ & $1.5 \pm 0.6$ & 100 & 100 \\
\hline $\begin{array}{l}\text { rApfA+rApxIA+rApxIIA+rApxIIIA } \\
+r A p x I V A+r T b p B\end{array}$ & 7 & $0.3 \pm 0.5^{7,8}$ & $39.6 \pm 0.5^{7}$ & $0.3 \pm 0.5^{7,8}$ & $29^{7}$ & 100 \\
\hline Control (PBS in oil adjuvant) & 7 & $3.2 \pm 0.7$ & $41.2 \pm 0.4$ & $2.3 \pm 0.7$ & 100 & 57 \\
\hline Non-treated & 2 & 0 & $39.1 \pm 0.4$ & 0 & 0 & 100 \\
\hline
\end{tabular}

${ }^{1}$ Pigs were immunized with two doses of the indicated vaccines at two week intervals and challenged intranasally two weeks later with A. pleuropneumoniae serotype 9 strain $\left(2 \times 10^{8} \mathrm{CFU}\right)$.

${ }^{2}$ Two independent immunization and challenge experiments were performed. Total number of pigs per vaccine group is given.

${ }^{3}$ Following the challenge, pigs were examined for clinical signs of pleuropneumonia and scored every day for seven days using a 0 to 4 scale. Mean value for each vaccination group calculated from two independent challenge experiments \pm standard deviation is given.

${ }^{4}$ Following the challenge, body temperature was taken daily for seven days. Mean value for each vaccination group calculated from two independent challenge experiments \pm standard deviation is given.

${ }^{5}$ Upon death, or immediately upon sacrifice on day seven, all animals underwent necropsy and macroscopic inspection of lungs. The observed lung lesions were scored using a 0 to 3 scale and mean value for each group calculated from two independent challenge experiments \pm standard deviation is given.

${ }^{6}$ Percentages of pigs in each vaccination group from which the challenge strain of A. pleuropneumoniae was re-isolated.

${ }^{7}$ Statistically significant difference $(P<0.02)$ between the vaccine immunized group and the control group, each comprising at least 7 animals.

${ }^{8}$ Statistically significant difference $(P<0.02)$ between the combined hexa-antigen vaccine ( $r A p f A$, rApxIA to IVA and rTbpB) immunized group and the pentaantigen vaccine ( $r A p x I A$ to IVA and $r T b p B$ ) immunized group.

lobes were scored separately and the mean lung lesion score was calculated for each animal. To check for presence of the challenge bacteria, the cranial lobe of each lung and lung portions exhibiting lesions were replica printed on a blood agar plate (Columbia Blood Agar Base, HIMEDIA, Mumbai, India) containing 6\% of sheep defibrinated blood. Colonies of A. pleuropneumoniae were grown for $48 \mathrm{~h}$ at $37^{\circ} \mathrm{C}$ in the hemolytic zone of Staphylococcus aureus Cowan I (CCM 2352; Veterinary Research Institute, Brno, Czech Republic). After incubation, the presence of satellite colonies was scored as suspect positive for A. pleuropneumoniae . Suspect colonies were confirmed as A. pleuropneumoniae serotype 9 strain by the PCR method and the coagulation test as previously described $[69,70]$.

\section{ELISA}

Wells of the PolySorp ELISA plates (Nunc, Roskilde, Denmark) were coated with $100 \mu \mathrm{L}$ per well of particular purified protein antigen in concentration of $32 \mathrm{ng} / \mathrm{mL}$ (rApfA), $63 \mathrm{ng} / \mathrm{mL}$ (rApxIA), $63 \mathrm{ng} / \mathrm{mL}$ (rApxIIA), 32 $\mathrm{ng} / \mathrm{mL}$ (rApxIIIA), $32 \mathrm{ng} / \mathrm{mL}$ (rApxIVA) and $750 \mathrm{ng} / \mathrm{mL}$ $(\mathrm{rTbpB})$, respectively. In addition, a mock extract of $E$. coli BL21( $\lambda \mathrm{DE} 3)$, processed by the same chromatographic procedure as the recombinant proteins, was also used for coating, in order to control the reaction of sera to any residual E. coli components within the protein preparations. Plates were coated overnight at $4^{\circ} \mathrm{C}$, washed three times with $300 \mu \mathrm{L}$ of PBS containing $0.05 \%$ Tween20 (Sigma-Aldrich, St. Louis, USA) (PBST) and blocked for $1 \mathrm{~h}$ with $1 \%$ BSA (Sigma-Aldrich, St. Louis, USA) in PBST. Serial dilutions of individual hyperimmune sera were added and the plates were incubated for $1 \mathrm{~h}$ at $37^{\circ} \mathrm{C}$. After rinsing with $\mathrm{PBST}$, peroxidase-conjugated rabbit anti-swine IgG (Amersham Biosciences, Piscataway, USA) was used at a 1:3000 dilution and the plates were incubated for $1 \mathrm{~h}$ at $37^{\circ} \mathrm{C}$. After washing with PBST and incubation with o-phenylenediamine peroxidase substrate solution, the reactions were stopped with $2 \mathrm{M}$ sulphuric acid and read at $492 \mathrm{~nm}$ using a Safire II microplate reader (Tecan, Zürich, Switzerland). The cutoff values for determination of anti-antigen (rApfA, rApxIA, rApxIIA, rApxIIIA, rApxIVA or rTbpB) IgG antibody titers were determined as the mean plus two standard deviations from the test results of preimmune sera at 1:100 dilution. The titers of antigen-specific antibodies were then defined as the reciprocal of the last dilution yielding an absorbance at $492 \mathrm{~nm}$ greater than the cutoff value.

\section{Statistical analysis}

The values of clinical signs, body temperatures, lung lesion scores and antibody titers were calculated from the data obtained for particular animals included in a given 
vaccination group and the numbers in the text, figures and tables thus represent mean values \pm standard deviations. To analyze the differences in various parameters among the vaccination groups, the Student's $t$-test was used on groups comprising at least 7 animals and the threshold for significance was set as $P<0.02$.

\section{Results}

\section{Cloning, expression and purification of recombinant antigens}

The genes coding for ApfA, ApxIA, ApxIIA, ApxIIIA, ApxIVA and TbpB were amplified from genomic DNA of A. pleuropneumoniae strains, cloned into an expression vector and overexpressed in $E$. coli cells. Since the production of the full-length ApfA protein was shown to be highly toxic for E. coli [64], ApfA lacking the N-terminal secretion signal and a predicted hydrophobic region (residues 1 to 35) was produced. The removal of the hydrophobic portion was expected to prevent the potential aggregation of the recombinant ApfA and the observed toxicity to $E$. coli cells [64]. All recombinant antigens were purified close to homogeneity from inclusion bodies by an affinity chromatography on Ni-NTA agarose. This procedure yielded per liter of bacterial culture approximately $5 \mathrm{mg}$ of rApfA, $40 \mathrm{mg}$ of rApxIA, rApxIIA or rApxIIIA, $15 \mathrm{mg}$ of rApxIVA and $50 \mathrm{mg}$ of rTbpB, respectively. Homogeneity of the final protein preparations was tested by SDS-PAGE (Figure 1) and the identity of the recombinant antigens was confirmed by MALDI-TOF mass spectrometry (data not shown).
Immunization with the ApfA antigen reduces clinical signs of porcine pleuropneumonia and significantly increases the potency of a multi-antigen vaccine

Prior to challenge, the pigs were randomly allocated into 8 groups and intramuscular immunization was performed with rApfA, rApxIVA or rTbpB individually, or with a triantigen combination of rApxIA to IIIA, or with a combined penta-antigen vaccine containing rApxIA to IVA and $r \operatorname{TbpB}$, or with a hexa-antigen vaccine including rApfA, rApxIA to IVA and rTbpB, or with PBS in oil adjuvant, respectively (Table 2). Animals in the last group were neither immunized nor challenged. Two independent immunization and challenge experiments were performed.

The post-vaccination sera from all piglets were taken one day before challenge and examined for anti-rApfA, anti-rApxIA to IVA and anti-rTbpB antibody levels using ELISA. As documented in Figure 2, the post-vaccination sera taken from all animals of a given vaccination group exhibited high titers (over 72 900) of antibodies against each of the individual component of the vaccine, but did not contain antibodies against an antigen that was not included in the vaccine. As further documented in Figure 2, no significant levels of rApfA, rApxIA to IVA or rTbpB specific antibodies were detected in sera of animals immunized with PBS in oil adjuvant or in sera of non-treated animals. As a control, preimmune sera were tested for the presence of anti-rApfA, anti-rApxIA to IVA and anti-rTbpB antibodies and were found to be negative (data not shown). Altogether, these results demonstrated that the rApfA, rApxIA to IVA and rTbpB

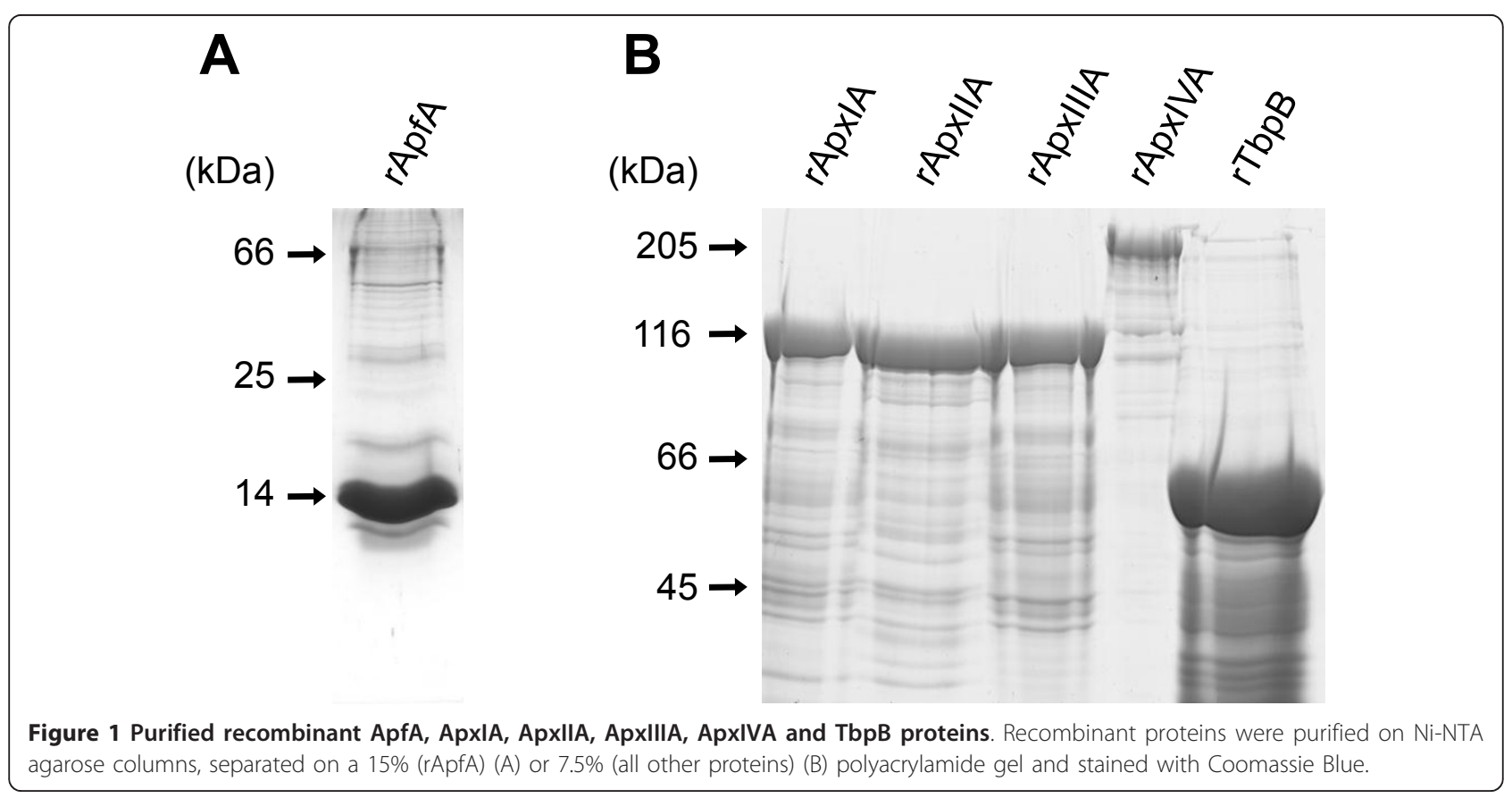




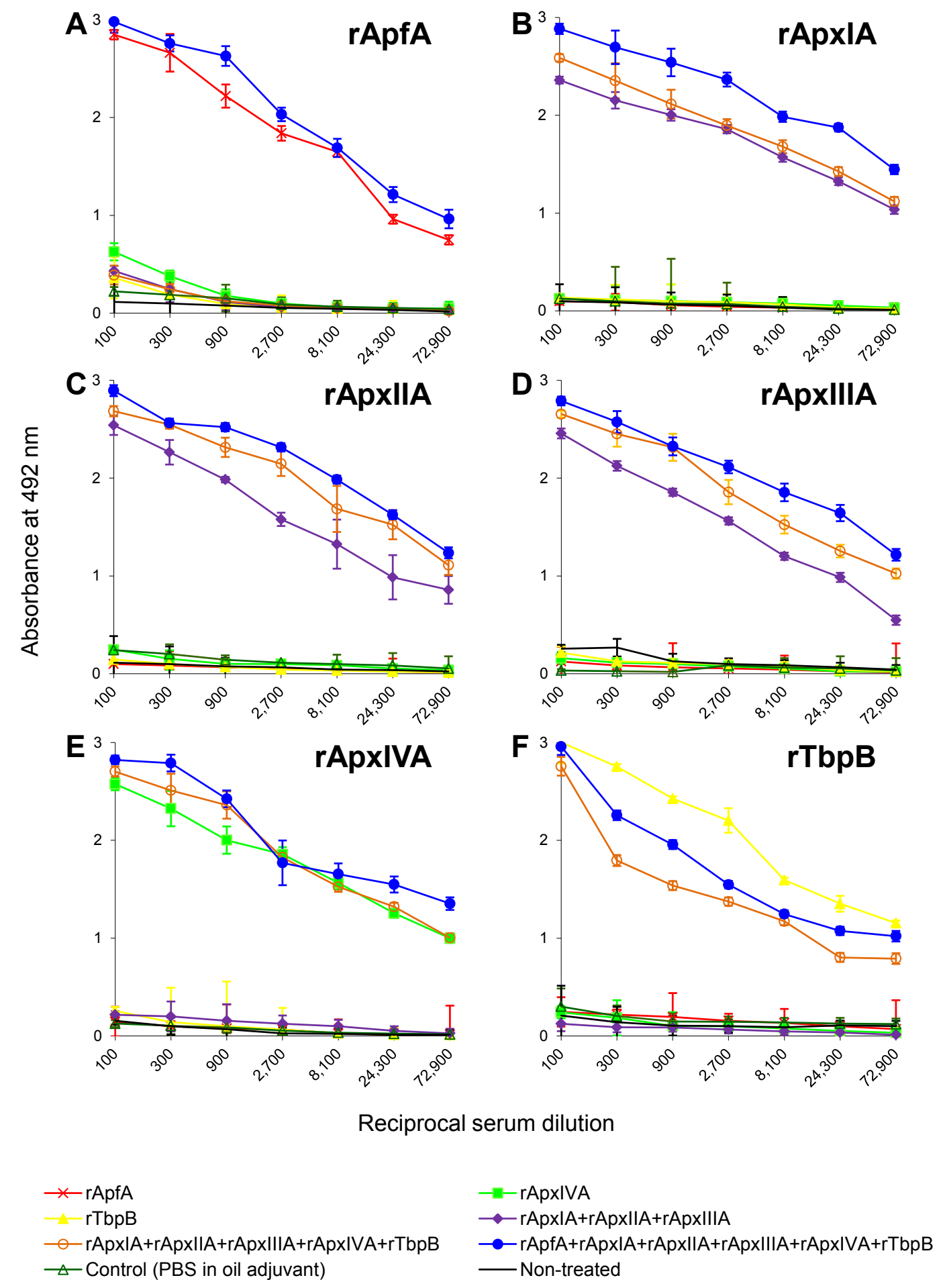

Figure 2 Titration of porcine post-vaccination sera. Pigs were immunized with two doses of the indicated vaccines at two week intervals and the post-vaccination sera from all piglets were taken one day before challenge and examined for the anti-rApfA (A), anti-rApxIA (B), antirApxIIA (C), anti-rApxIIIA (D), anti-rApxIVA (E) and anti-rTbpB (F) antibody levels using ELISA. Each point represents an average value calculated from results obtained for titration of sera taken from all pigs included in the given vaccination group \pm standard deviation. 
proteins were highly immunogenic and induced specific serum antibodies.

The protective potency of the vaccines was tested in heterologous challenge experiments using an infectious dose of $2 \times 10^{8} \mathrm{CFU}$ of A. pleuropneumoniae serotype 9 strain that was applied via the intranasal route one month after the first immunization. In the control group of animals that received PBS in oil adjuvant, all pigs suffered from severe cough, dyspnea, apathy and fever fluctuating between $40.8-41.6^{\circ} \mathrm{C}$ within seven days following the challenge with $A$. pleuropneumoniae serotype 9 strain (Table 2). Two out of seven pigs died within $24 \mathrm{~h}$ after the challenge and one animal was found dead on the second day after inoculation, while the remaining pigs survived until the end of the study, despite severe clinical signs of porcine pleuropneumonia. Upon necropsy, all pigs in this group showed lung lesions characterized by a hemorrhagic necrotizing pneumonia and fibrinous pleuritis typical of $A$. pleuropneumoniae infection with the mean lung lesion score $2.3 \pm 0.7$ out of 3 (Table 2). The challenge strain was re-isolated from the lungs of all pigs, confirming the etiology of the lesions (Table 2). These results demonstrated that the challenge with the heterologous A. pleuropneumoniae serotype 9 strain caused typical signs of severe porcine pleuropneumonia.

In contrast to animals that received only PBS in oil adjuvant, the pigs vaccinated with rApfA showed considerably milder clinical signs of porcine pleuropneumonia (Table 2 ). The animals suffered from coughing, increased respiration rate and mild apathy for seven days after the challenge and fever up to $40.8^{\circ} \mathrm{C}$. Corresponding to milder clinical signs of pleuropneumonia, also the mean lung lesion score was significantly lower in animals immunized with rApfA $(1.3 \pm 0.7, P<0.02)$, when compared to the control $(2.3 \pm 0.7)$ (Table 2). The challenge strain was reisolated from the lungs of seven out of twelve animals (Table 2).

Immunization with rApxIVA or rTbpB alone or with the tri-antigen combination of rApxIA to IIIA, respectively, did not protect the challenged animals against development of clinical signs of the disease, however, none of the pigs died within seven days following the challenge (Table 2). Upon challenge, the animals immunized with rApxIVA or $\mathrm{rTbpB}$ alone suffered mainly from cough, abdominal respiration, dyspnea, apathy and fever up to $41.4^{\circ} \mathrm{C}$. Upon necropsy, a massive destruction of the lung tissue was observed (Table 2). On the contrary, the pigs vaccinated with the tri-antigen combination of rApxIA to IIIA showed milder clinical signs of porcine pleuropneumonia and lower mean lung lesion score than the animals vaccinated with rApxIVA or rTbpB alone (Table 2). The challenge strain was recovered from the lungs of all pigs vaccinated with rApxIVA or $\mathrm{rTbpB}$ alone or with the tri-antigen combination, respectively (Table 2 ).

All these data demonstrate that rApfA alone provided a partial protection against infection with $A$. pleuropneumoniae that was higher than the protection conferred by the immunization with rApxIVA or $\mathrm{rTbpB}$ alone and similar to the protection after vaccination with the triantigen combination of rApxIA, rApxIIA and rApxIIIA.

When the purified rApxIA to IVA and rTbpB proteins were combined in a penta-antigen vaccine and used for immunization, a high level of protection against the challenge with $A$. pleuropneumoniae serotype 9 strain was observed (Table 2). Pigs mainly suffered from increased respiration rate, coughing, occasional lying down and mild fever not exceeding $40.3^{\circ} \mathrm{C}$. The observed clinical signs had a tendency to diminish over the seven day period after the challenge. The mean lung lesion score was significantly lower in animals immunized with the combined penta-antigen vaccine $(1.0 \pm$ $0.6, P<0.02)$, when compared to the control group $(2.3 \pm 0.7)$ (Table 2$)$. The challenge strain was re-isolated from the lungs of six out of seven pigs included in this experimental group (Table 2). These data indicate that the penta-antigen vaccine provided high level of protection against acute disease upon challenge with A. pleuropneumoniae .

When this penta-antigen vaccine was supplemented with the purified rApfA and used for immunization, the combined hexa-antigen vaccine containing rApfA, rApxIA to IVA and $\mathrm{rTbpB}$, respectively, conferred on animals a high level of protection against all clinical signs accompanying acute infection (Table 2). Pigs showed only sporadic coughing and occasional lying down for the first three days and presented mild fever about $40.1^{\circ} \mathrm{C}$ during the first two days after the challenge. After the period of three days, none of the animals showed any clinical signs of the disease or fever. Following necropsy, none or only minor pathological changes were observed in the lungs of these animals and the mean lung lesion score was $0.3 \pm 0.5$ (Table 2), which was fully in line with the absence of signs. The challenge strain was isolated from the lungs of only two out of the seven challenged animals (Table 2). These results demonstrate that rApfA further improved the vaccine potency of the currently used antigens and that the immunization with the combined hexa-antigen vaccine consisting of rApfA, rApxIA to IVA and rTbpB enabled a high level of protection of pigs against pleuropneumonia. Moreover, rApfA used alone or in combination with other antigens significantly decreased the number of animals colonized by the heterologous challenge strain of A. pleuropneumoniae. Hence, ApfA should be included in a new generation of subunit vaccines against porcine pleuropneumonia, providing not only a complete protection of 
pigs against the clinical signs of the acute disease, but also limiting lung colonization by $A$. pleuropneumoniae .

\section{Discussion}

To develop more effective subunit vaccines against porcine pleuropneumonia, the identification, production and testing of novel $A$. pleuropneumoniae protein antigens are the objectives of current research. We show here that the type IV fimbrial subunit protein ApfA should be considered as a promising vaccine candidate.

We demonstrate that vaccination of pigs with the recombinant ApfA protein alone provided limited protection against heterologous challenge with A. pleuropneumoniae. This result was in agreement with previous reports showing that single antigen vaccines offer only partial or no protection against pleuropneumonia. For instance, when ApxIIA and TbpB proteins expressed in E. coli were used for vaccination separately, all pigs developed a strong humoral immune response and upon challenge with a homologous $A$. pleuropneumoniae serotype 7 strain, all immunized animals were less affected by the pleuropneumonia and demonstrated significantly lower mortality than the controls [53]. However, protection was serotypespecific, since no cross-protection was detected, when the animals were challenged with an $A$. pleuropneumoniae serotype 1 strain [53]. Recently, the N-terminal portion of ApxIVA produced in E. coli was used for vaccination and the challenged pigs showed similar severe respiratory signs and severe lung lesions as the animals in the control group, despite high antibody titers against the $\mathrm{N}$-terminus of ApxIVA [49]. We obtained similar results showing that the vaccination of pigs with full-length rApxIVA or rTbpB alone protected animals against death upon heterologous challenge with $A$. pleuropneumoniae serotype 9 strain, but did not protect them against development of clinical signs of the disease and massive destruction of the lung tissue, despite high titers of specific antibodies. In contrast, the pigs immunized with the recombinant ApfA alone showed milder clinical signs of porcine pleuropneumonia and reduced destruction of the lung tissue than the animals vaccinated with rApxIVA or rTbpB alone.

Since single antigens often provide only limited protection against porcine pleuropneumonia, multi-antigen subunit vaccines were used for immunization. For instance, pigs receiving a mixture of the recombinant ApxIIA and TbpB proteins had a tendency to recover faster than animals that were vaccinated with only one antigen [53]. Similarly, the N-terminal portion of ApxIVA improved protective immunity conferred by the subunit vaccine containing recombinant ApxIA, ApxIIA, ApxIIIA and the 42$\mathrm{kDa}$ outer membrane protein [49]. Therefore, we also tested whether recombinant ApfA would improve protection upon its addition to a multi-antigen subunit vaccine. Our data demonstrate that the immunization of pigs with the combined hexa-antigen vaccine consisting of rApfA, rApxIA to IVA and $\mathrm{rTbpB}$ provided protective immunity against pleuropneumonia that was significantly higher than that acquired upon vaccination with rApfA, rApxIVA or $r \mathrm{TbpB}$ alone, with the tri-antigen combination of rApxIA, rApxIIA and rApxIIIA or even with the penta-antigen subunit vaccine composed of rApxIA to IVA and rTbpB. Thus, rApfA improved the vaccination potential of currently used antigens. Moreover, immunization of pigs with the combined hexa-antigen vaccine provided almost complete protection of animals against the heterologous challenge with A. pleuropneumoniae serotype 9 strain.

Recently, the contribution of recombinant ApfA to the protective immunity of a subunit vaccine comprising rApxIA, rApxIIA, rApxIIIA and rOMP was tested in mice [71]. It has been shown that the antibody titers against rApxIA, rApxIIA, rApxIIIA and rOMP were significantly lower in sera of mice immunized with the vaccine containing rApfA, rApxIA to IIIA and rOMP than the titers in sera of mice immunized with the vaccine comprising only rApxIA to IIIA and rOMP. In agreement, upon challenge with A. pleuropneumoniae, mice immunized with the vaccine containing rApfA, rApxIA to IIIA and rOMP had the lower survival rate and more severe lung lesions than the animals immunized with the vaccine lacking rApfA. Thus, it has been suggested that vaccination with rApfA may impair immunity of mice [71], similarly as described for the outer membrane protein PalA of A pleuropneumoniae in pigs [68]. Our immunization and challenge experiments in pigs, however, do not support the observation achieved with rApfA in mice. As documented in Figure 2, we clearly show that the post-vaccination sera of pigs immunized with the hexa-antigen vaccine containing rApfA, rApxIA to IVA and rTbpB exhibited higher or comparable levels of rApxIA, rApxIIA or rApxIIIA specific antibodies than the sera of animals immunized with the penta-antigen vaccine comprising only rApxIA to IVA and rTbpB. In addition, the hexa-antigen vaccine conferred on pigs a significantly better protection against pleuropneumonia than did the penta-antigen vaccine lacking rApfA (Table 2).

Type IV fimbriae are known to play a key role in virulence of many species of Gram-negative bacteria and therefore type IV fimbrial subunit proteins have been tested as subunit vaccines for the prevention of several diseases. For instance, a highly purified fimbriae prepared from cells of Dichelobacter nodosus provided a high level of protection against ovine foot-rot upon homologous challenge and lower levels of protection against challenge with strains of different serogroups [72]. Similarly, type IV fimbriae isolated and purified from different strains of Moraxella bovis effectively protected cattle against experimentally induced infectious bovine keratoconjunctivitis upon homologous challenge, but provided only partial protection against challenge with a strain of different 
serogroup [73]. Moreover, a change in pilus antigenicity by pilin gene inversion enabling bacterial escape from protective immunity was suggested to occur in response to the presence of pilin-specific antibodies upon vaccination with purified fimbriae of $M$. bovis [74]. The antigenic variation of the pilin subunit has also been an important obstacle to the development of an effective pilus-based vaccine for Neisseria gonorrhoeae [75].

We demonstrate here that despite high antibody titers against rApfA, vaccination of pigs with rApfA alone provided only partial protection against heterologous challenge with A. pleuropneumoniae. However, the apfA alleles were found to be highly conserved and present in a single copy in the genomes of different A. pleuropneumoniae serotype strains [18]. Therefore, the observed partial protection of immunized animals against pleuropneumonia was most probably not due to the low level of the ApfA sequence homology or the pilin antigenic variation in the challenge strain. A more plausible explanation is that some additional components to type IV fimbriae are involved in adherence of A. pleuropneumoniae to alveolar epithelial cells, which is an important initial step in the pathogenesis of porcine pleuropneumoniae $[20,76]$. Indeed, A. pleuropneumoniae was shown to bind to phosphatidylethanolamine of the host cells through $\mathrm{O}$-antigen of its cell-wall lipopolysaccharides [77]. Moreover, a 55$\mathrm{kDa}$ outer membrane protein (OMP) was shown to play a role in the adherence of $A$. pleuropneumoniae to alveolar epithelial cells in culture [78]. Thus, a multiple-step binding process has been suggested, in which $A$. pleuropneumoniae first uses low-affinity binding between $\mathrm{O}$-antigen and phospholipids or short glycolipids and then relies on the core oligosaccharide of LPS and/or surface proteins, such as $55-\mathrm{kDa}$ OMP and type IV fimbriae, to interact more avidly with other larger lipidic and/or proteinaceous receptors on host cells [77]. The use of multiple parallel adhesion mechanisms by $A$. pleuropneumoniae and involvement of additional virulence factors would, hence, provide a likely explanation for the observed only partial protective efficacy of the vaccine based solely on ApfA, which was not able to completely prevent the colonization of the lungs of pigs by the challenge strain of A. pleuropneumoniae . Conversely, the reduction of the number of pigs colonized by the challenge strain up to $29 \%$ upon immunization with the ApfA-based vaccines suggests that ApfA-specific antibodies might markedly inhibit the pilusmediated binding of A. pleuropneumoniae to host cells, despite the existence of other adherence mechanisms.

In conclusion, our results demonstrate that immunization of pigs with the type IV fimbrial subunit protein ApfA induced specific serum antibody response that reduced clinical signs of the acute disease upon heterologous challenge with $A$. pleuropneumoniae and significantly increased the potency of the currently used protective antigens. Thus, ApfA could be a candidate to be included in a next generation of subunit vaccines against porcine pleuropneumonia.

\section{Acknowledgements}

This work was supported by research and development project Tandem FT TA2/025 from the Ministry of Industry and Trade (JN and W), grant KAN200520702 of the Grant Agency of the Academy of Sciences of the Czech Republic (RO and LS), grants P302/11/0580 (RO) and P207/11/0717 (PS) of the Czech Science Foundation and Institutional Research Concept AVOZ50200510 (RO and PS).

\section{Author details}

${ }^{1}$ Institute of Microbiology of the Academy of Sciences of the Czech Republic, v.v.i., Videnska 1083, CZ-142 20 Prague, Czech Republic. ${ }^{2}$ Bioveta, a. s., Komenskeho 212/12, CZ-683 23 Ivanovice na Hane, Czech Republic.

\section{Authors' contributions}

LS participated in the design of the study, performed vector construction, protein expression, purification and analysis, carried out ELISA experiments, analyzed data and drafted the manuscript. JN participated in the design of the study and in the development of ELISA protocols, performed immunizations and challenge experiments in pigs and collected and analyzed data. W conceived of the study, participated in its design and coordination. PS participated in the design of the study and edited the manuscript. RO participated in the design of the study, in the development of ELISA protocols and protein expression and purification methods and edited the manuscript. All authors read and approved the final manuscript.

\section{Competing interests}

The authors declare that they have no competing interests.

Received: 16 August 2011 Accepted: 12 January 2012

Published: 12 January 2012

\section{References}

1. Gottschalk M, Taylor DJ: Actinobacillus pleuropneumoniae. In Diseases of Swine.. 9 edition. Edited by: Straw BE, Zimmerman JJ, D'Allaire S, Taylor DJ. Ames, lowa: Blackwell Publishing; 2006:563-576.

2. Sebunya TN, Saunders JR: Haemophilus pleuropneumoniae infection in swine: a review. J Am Vet Med Assoc 1983, 182:1331-1337.

3. Chiers K, De Waele T, Pasmans F, Ducatelle R, Haesebrouck F: Virulence factors of Actinobacillus pleuropneumoniae involved in colonization, persistence and induction of lesions in its porcine host. Vet Res 2010, 41:65.

4. Bosse JT, Janson H, Sheehan BJ, Beddek AJ, Rycroft AN, Kroll JS, Langford PR: Actinobacillus pleuropneumoniae : pathobiology and pathogenesis of infection. Microbes Infect 2002, 4:225-235.

5. Rosendal S, Boyd DA, Gilbride KA: Comparative virulence of porcine Haemophilus bacteria. Can J Comp Med 1985, 49:68-74.

6. Shope RE, White DC, Leidy G: Porcine contagious Pleuropneumonia. II. Studies of the pathogenicity of the etiological agent, Hemophilus pleuropneumoniae. J Exp Med 1964, 119:369-375.

7. Liggett $A D$, Harrison LR, Farrell RL: Sequential study of lesion development in experimental haemophilus pleuropneumonia. Res Vet Sci 1987, 42:204-212.

8. Sanford SE, Josephson GK: Porcine Haemophilus pleuropneumonia epizootic in southwestern Ontario: clinical, microbiological, pathological and some epidemiological findings. Can J Comp Med 1981, 45:2-7.

9. Jones MA: Haemophilus pleuropneumoniae infection in pigs. In The veterinary annual. Edited by: Grunsell CSG, Hill FWG. Bristol, England: John Wright and Sons Ltd; 1984:, 24: 148-154

10. Haesebrouck F, Pasmans F, Chiers K, Maes D, Ducatelle R, Decostere A: Efficacy of vaccines against bacterial diseases in swine: what can we expect? Vet Microbiol 2004, 100:255-268.

11. Ramjeet M, Deslandes V, Goure J, Jacques M: Actinobacillus pleuropneumoniae vaccines: from bacterins to new insights into vaccination strategies. Anim Health Res Rev 2008, 9:25-45. 
12. Blackall PJ, Klaasen HL, van den Bosch H, Kuhnert P, Frey J: Proposal of a new serovar of Actinobacillus pleuropneumoniae : serovar 15. Vet Microbiol 2002, 84:47-52.

13. Dubreuil JD, Jacques M, Mittal KR, Gottschalk M: Actinobacillus pleuropneumoniae surface polysaccharides: their role in diagnosis and immunogenicity. Anim Health Res Rev 2000, 1:73-93.

14. Jacques M: Surface polysaccharides and iron-uptake systems of Actinobacillus pleuropneumoniae . Can J Vet Res 2004, 68:81-85.

15. Higgins R, Lariviere S, Mittal KR, Martineau GP, Rousseau P, Cameron J: Evaluation of a killed vaccine against porcine pleuropneumonia due to Haemophilus pleuropneumoniae . Can Vet J 1985, 26:86-89.

16. Nielsen R: Haemophilus pleuropneumoniae serotypes-cross protection experiments. Nord Vet Med 1984, 36:221-234.

17. Nielsen R: Haemophilus pleuropneumoniae (Actinobacillus pleuropneumoniae ). Serotypes 8, 3 and 6. Serological response and cross immunity in pigs. Nord Vet Med 1985, 37:217-227.

18. Boekema BK, Van Putten JP, Stockhofe-Zurwieden N, Smith HE: Host cell contact-induced transcription of the type IV fimbria gene cluster of Actinobacillus pleuropneumoniae . Infect Immun 2004, 72:691-700.

19. Cho WS, Chae C: Expression of the apxIV gene in pigs naturally infected with Actinobacillus pleuropneumoniae. J Comp Pathol 2001, 125:34-40.

20. Haesebrouck F, Chiers K, Van Overbeke I, Ducatelle R: Actinobacillus pleuropneumoniae infections in pigs: the role of virulence factors in pathogenesis and protection. Vet Microbiol 1997, 58:239-249.

21. Haga Y, Ogino S, Ohashi S, Ajito T, Hashimoto K, Sawada T: Protective efficacy of an affinity-purified hemolysin vaccine against experimental swine pleuropneumonia. J Vet Med Sci 1997, 59:115-120.

22. Seah JN, Frey J, Kwang J: The N-terminal domain of RTX toxin Apxl of Actinobacillus pleuropneumoniae elicits protective immunity in mice. Infect Immun 2002, 70:6464-6467.

23. Cruijsen T, van Leengoed LA, Ham-Hoffies M, Verheijden JH: Convalescent pigs are protected completely against infection with a homologous Actinobacillus pleuropneumoniae strain but incompletely against a heterologous-serotype strain. Infect Immun 1995, 63:2341-2343.

24. Haesebrouk F, Van de Kerkhof A, Dom P, Chiers K, Ducatelle R: Crossprotection between Actinobacillus pleuropneumoniae biotypes-serotypes in pigs. Vet Microbiol 1996, 52:277-284.

25. Baltes N, Tonpitak W, Gerlach GF, Hennig-Pauka I, Hoffmann-Moujahid A, Ganter M, Rothkotter HJ: Actinobacillus pleuropneumoniae iron transport and urease activity: effects on bacterial virulence and host immune response. Infect Immun 2001, 69:472-478.

26. Byrd W, Hooke AM: Immunization with temperature-sensitive mutants of Actinobacillus pleuropneumoniae induces protective hemolysinneutralizing antibodies in mice. Curr Microbiol 1997, 34:149-154.

27. Fuller TE, Thacker BJ, Mulks MH: A riboflavin auxotroph of Actinobacillus pleuropneumoniae is attenuated in swine. Infect Immun 1996, 64:4659-4664

28. Garside LH, Collins M, Langford PR, Rycroft AN: Actinobacillus pleuropneumoniae serotype 1 carrying the defined aroA mutation is fully avirulent in the pig. Res Vet Sci 2002, 72:163-167.

29. Ingham A, Zhang Y, Prideaux C: Attenuation of Actinobacillus pleuropneumoniae by inactivation of aroQ. Vet Microbiol 2002, 84:263-273.

30. Maas A, Jacobsen ID, Meens J, Gerlach GF: Use of an Actinobacillus pleuropneumoniae multiple mutant as a vaccine that allows differentiation of vaccinated and infected animals. Infect Immun 2006, 74:4124-4132.

31. Prideaux $C T$, Lenghaus $C$, Krywult J, Hodgson AL: Vaccination and protection of pigs against pleuropneumonia with a vaccine strain of Actinobacillus pleuropneumoniae produced by site-specific mutagenesis of the Apxll operon. Infect Immun 1999, 67:1962-1966.

32. Prideaux $C T$, Pierce $L$, Krywult J, Hodgson AL: Protection of mice against challenge with homologous and heterologous serovars of Actinobacillus pleuropneumoniae after live vaccination. Curr Microbiol 1998, 37:324-332.

33. Tumamao JQ, Bowles RE, van den Bosch H, Klaasen HL, Fenwick BW, Storie GJ, Blackall PJ: Comparison of the efficacy of a subunit and a live streptomycin-dependent porcine pleuropneumonia vaccine. Aust Vet $J$ 2004, 82:370-374

34. Xu F, Chen X, Shi A, Yang B, Wang J, Li Y, Guo X, Blackall PJ, Yang H: Characterization and immunogenicity of an apxIA mutant of Actinobacillus pleuropneumoniae. Vet Microbiol 2006, 118:230-239.
35. Bei W, He Q, Zhou R, Yan L, Huang H, Chen H: Evaluation of immunogenicity and protective efficacy of Actinobacillus pleuropneumoniae $\mathrm{HB} 04 \mathrm{C}(-)$ mutant lacking a drug resistance marker in the pigs. Vet Microbiol 2007, 125:120-127.

36. Lin L, Bei W, Sha Y, Liu J, Guo Y, Liu W, Tu S, He Q, Chen H: Construction and immunogencity of a DeltaapxIC/DeltaapxIIC double mutant of Actinobacillus pleuropneumoniae serovar 1. FEMS Microbiol Lett 2007, 274:55-62

37. Frey J, Meier R, Gygi D, Nicolet J: Nucleotide sequence of the hemolysin I gene from Actinobacillus pleuropneumoniae . Infect Immun 1991, 59:3026-3032.

38. Frey J: Virulence in Actinobacillus pleuropneumoniae and RTX toxins. Trends Microbiol 1995, 3:257-261.

39. Frey J, Beck M, Stucki U, Nicolet J: Analysis of hemolysin operons in Actinobacillus pleuropneumoniae. Gene 1993, 123:51-58.

40. Frey J, Beck M, van den Bosch JF, Segers RP, Nicolet J: Development of an efficient PCR method for toxin typing of Actinobacillus pleuropneumoniae strains. Mol Cell Probes 1995, 9:277-282.

41. Frey J, Bosse JT, Chang YF, Cullen JM, Fenwick B, Gerlach GF, Gygi D, Haesebrouck F, Inzana TJ, Jansen R, Kamp M, Macdonald J, MacInnes Jl, Mittal KR, Nicolet J, Rycroft AN, Segers RP, Smits MA, Stenbaek E, Struck DK, van den Bosch, Willson PJ, Young R: Actinobacillus pleuropneumoniae RTXtoxins: uniform designation of haemolysins, cytolysins, pleurotoxin and their genes. J Gen Microbiol 1993, 139:1723-1728.

42. Linhartova I, Bumba L, Masin J, Basler M, Osicka R, Kamanova J, Prochazkova K, Adkins I, Hejnova-Holubova J, Sadilkova L, Morová J, Sebo P: RTX proteins: a highly diverse family secreted by a common mechanism. FEMS Microbiol Rev 2010, 34:1076-1112.

43. Frey J, Nicolet J: Hemolysin patterns of Actinobacillus pleuropneumoniae . J Clin Microbiol 1990, 28:232-236.

44. Kamp EM, Popma JK, Anakotta J, Smits MA: Identification of hemolytic and cytotoxic proteins of Actinobacillus pleuropneumoniae by use of monoclonal antibodies. Infect Immun 1991, 59:3079-3085.

45. Rosendal S, Devenish J, Maclnnes JI, Lumsden JH, Watson S, Xun H: Evaluation of heat-sensitive, neutrophil-toxic, and hemolytic activity of Haemophilus (Actinobacillus ) pleuropneumoniae. Am J Vet Res 1988, 49:1053-1058.

46. Rycroft AN, Williams D, Cullen JM, Macdonald J: The cytotoxin of Actinobacillus pleuropneumoniae (pleurotoxin) is distinct from the haemolysin and is associated with a $120 \mathrm{kDa}$ polypeptide. J Gen Microbiol 1991, 137:561-568.

47. Chiers K, van Overbeke I, De Laender P, Ducatelle R, Carel S, Haesebrouck F: Effects of endobronchial challenge with Actinobacillus pleuropneumoniae serotype 9 of pigs vaccinated with inactivated vaccines containing the Apx toxins. Vet Q 1998, 20:65-69.

48. Van Overbeke I, Chiers K, Ducatelle R, Haesebrouck F: Effect of endobronchial challenge with Actinobacillus pleuropneumoniae serotype 9 of pigs vaccinated with a vaccine containing Apx toxins and transferrin-binding proteins. J Vet Med B Infect Dis Vet Public Health 2001 48:15-20.

49. Wang $C$, Wang $Y$, Shao M, Si W, Liu H, Chang $Y$, Peng W, Kong $X$, Liu S: Positive role for rApxIVN in the immune protection of pigs against infection by Actinobacillus pleuropneumoniae. Vaccine 2009, 27:5816-5821.

50. Schaller A, Kuhn R, Kuhnert P, Nicolet J, Anderson TJ, Maclnnes IJ, Segers RP, Frey J: Characterization of apxIVA, a new RTX determinant of Actinobacillus pleuropneumoniae. Microbiology 1999, 145:2105-2116.

51. Gonzalez GC, Caamano DL, Schryvers AB: Identification and characterization of a porcine-specific transferrin receptor in Actinobacillus pleuropneumoniae. Mol Microbiol 1990, 4:1173-1179.

52. Baltes N, Hennig-Pauka I, Gerlach GF: Both transferrin binding proteins are virulence factors in Actinobacillus pleuropneumoniae serotype 7 infection. FEMS Microbiol Lett 2002, 209:283-287.

53. Rossi-Campos A, Anderson C, Gerlach GF, Klashinsky S, Potter AA, Willson PJ: Immunization of pigs against Actinobacillus pleuropneumoniae with two recombinant protein preparations. Vaccine 1992, 10:512-518.

54. Oldfield NJ, Donovan EA, Worrall KE, Wooldridge KG, Langford PR, Rycroft AN, Ala'Aldeen DA: Identification and characterization of novel antigenic vaccine candidates of Actinobacillus pleuropneumoniae Vaccine 2008, 26:1942-1954. 
55. Zhang Y, Tennent JM, Ingham A, Beddome G, Prideaux C, Michalski WP: Identification of type 4 fimbriae in Actinobacillus pleuropneumoniae . FEMS Microbiol Lett 2000, 189:15-18.

56. Bakaletz LO, Baker BD, Jurcisek JA, Harrison A, Novotny LA, Bookwalter JE, Mungur R, Munson RS Jr: Demonstration of Type IV pilus expression and a twitching phenotype by Haemophilus influenzae. Infect Immun 2005, 73:1635-1643.

57. Luke NR, Howlett AJ, Shao J, Campagnari AA: Expression of type IV pili by Moraxella catarrhalis is essential for natural competence and is affected by iron limitation. Infect Immun 2004, 72:6262-6270.

58. Bieber D, Ramer SW, Wu CY, Murray WJ, Tobe T, Fernandez R, Schoolnik GK: Type IV pili, transient bacterial aggregates, and virulence of enteropathogenic Escherichia coli . Science 1998, 280:2114-2118.

59. Carbonnelle E, Helaine $S$, Nassif X, Pelicic V: A systematic genetic analysis in Neisseria meningitidis defines the Pil proteins required for assembly, functionality, stabilization and export of type IV pili. Mol Microbiol 2006, 61:1510-1522.

60. Koga T, Ishimoto K, Lory S: Genetic and functional characterization of the gene cluster specifying expression of Pseudomonas aeruginosa pili. Infect Immun 1993, 61:1371-1377.

61. Wall D, Kaiser D: Type IV pili and cell motility. Mol Microbio/ 1999, 32:1-10.

62. Craig L, Pique ME, Tainer JA: Type IV pilus structure and bacterial pathogenicity. Nat Rev Microbiol 2004, 2:363-378.

63. Hansen JK, Forest KT: Type IV pilin structures: insights on shared architecture, fiber assembly, receptor binding and type II secretion. J Mol Microbiol Biotechnol 2006, 11:192-207.

64. Stevenson A, Macdonald J, Roberts M: Cloning and characterisation of type 4 fimbrial genes from Actinobacillus pleuropneumoniae. Vet Microbiol 2003, 92:121-134.

65. Sambrook J, Fritsch EF, Maniatis T: Molecular cloning: a laboratory manual. Cold Spring Harbor Laboratory Press, Cold Spring Harbor, N.Y., USA; 21989.

66. Foote SJ, Bosse JT, Bouevitch AB, Langford PR, Young NM, Nash JH: The complete genome sequence of Actinobacillus pleuropneumoniae L20 (serotype 5b). J Bacteriol 2008, 190:1495-1496.

67. Xu Z, Zhou Y, Li L, Zhou R, Xiao S, Wan Y, Zhang S, Wang K, Li W, Jin H, Kang M, Dalai B, Li T, Liu L, Cheng Y, Zhang L, Xu T, Zheng H, Pu S, Wang B, Gu W, Zhang XL, Zhu GF, Wang S, Zhao GP, Chen H: Genome biology of Actinobacillus pleuropneumoniae JL03, an isolate of serotype 3 prevalent in China. PLoS One 2008, 3:e1450.

68. van den Bosch $\mathrm{H}$, Frey J: Interference of outer membrane protein PalA with protective immunity against Actinobacillus pleuropneumoniae infections in vaccinated pigs. Vaccine 2003, 21:3601-3607.

69. Kucerova Z, Jaglic Z, Ondriasova R, Nedbalcova K: Serotype distribution of Actinobacillus pleuropneumoniae isolated from porcine pleuropneumonia in the Czech Republic during period 2003-2004. Veterinarni Medicina UZPI (Czech Republic) 2005, 50:355-360.

70. Mittal KR, Higgins R, Lariviere S: Identification and serotyping of Haemophilus pleuropneumoniae by coagglutination test. J Clin Microbiol 1983, 18:1351-1354.

71. Shao M, Wang Y, Wang C, Guo Y, Peng Y, Liu J, Li G, Liu H, Liu S: Evaluation of multicomponent recombinant vaccines against Actinobacillus pleuropneumoniae in mice. Acta Vet Scand 2010, 52:52.

72. Stewart DJ, Clark BL, Peterson JE, Emery DL, Smith EF, Griffiths DA, O'Donnell IJ: The protection given by pilus and whole cell vaccines of Bacteroides nodosus strain 198 against ovine foot-rot induced by strains of different serogroups. Aust Vet J 1985, 62:153-159.

73. Lepper AW, Moore LJ, Atwell JL, Tennent JM: The protective efficacy of pili from different strains of Moraxella bovis within the same serogroup against infectious bovine keratoconjunctivitis. Vet Microbiol 1992, 32:177-187.

74. Lepper AW, Atwell JL, Lehrbach PR, Schwartzkoff CL, Egerton JR, Tennent JM: The protective efficacy of cloned Moraxella bovis pili in monovalent and multivalent vaccine formulations against experimentally induced infectious bovine keratoconjunctivitis (IBK). Vet Microbiol 1995, 45:129-138.

75. Boslego JW, Tramont EC, Chung RC, McChesney DG, Ciak J, Sadoff JC, Piziak MV, Brown JD, Brinton CC, Wood SW, Bryan JR: Efficacy trial of a parenteral gonococcal pilus vaccine in men. Vaccine 1991, 9:154-162.

76. Dom P, Haesebrouck F, Ducatelle R, Charlier G: In vivo association of Actinobacillus pleuropneumoniae serotype 2 with the respiratory epithelium of pigs. Infect Immun 1994, 62:1262-1267.
77. Jeannotte ME, Abul-Milh M, Dubreuil JD, Jacques M: Binding of Actinobacillus pleuropneumoniae to phosphatidylethanolamine. Infect Immun 2003, 71:4657-4663.

78. Van Overbeke I, Chiers K, Charlier G, Vandenberghe I, Van Beeumen J, Ducatelle R, Haesebrouck F: Characterization of the in vitro adhesion of Actinobacillus pleuropneumoniae to swine alveolar epithelial cells. Vet Microbiol 2002, 88:59-74.

doi:10.1186/1297-9716-43-2

Cite this article as: Sadilkova et al:: Type IV fimbrial subunit protein ApfA contributes to protection against porcine pleuropneumonia. Veterinary Research 2012 43:2.

\section{Submit your next manuscript to BioMed Central and take full advantage of:}

- Convenient online submission

- Thorough peer review

- No space constraints or color figure charges

- Immediate publication on acceptance

- Inclusion in PubMed, CAS, Scopus and Google Scholar

- Research which is freely available for redistribution

Submit your manuscript at www.biomedcentral.com/submit
Biomed Central 